PROCEEDINGS OF THE

AMERICAN MATHEMATICAL SOCIETY

Volume 125, Number 4, April 1997, Pages 1043-1054

S 0002-9939(97)03611-3

\title{
HYPERCOMPLEX STRUCTURES ON FOUR-DIMENSIONAL LIE GROUPS
}

\author{
MARÍA LAURA BARBERIS
}

(Communicated by Roe Goodman)

\begin{abstract}
The purpose of this paper is to classify invariant hypercomplex structures on a 4-dimensional real Lie group $G$. It is shown that the 4dimensional simply connected Lie groups which admit invariant hypercomplex structures are the additive group $\mathbb{H}$ of the quaternions, the multiplicative group $\mathbb{H}^{*}$ of nonzero quaternions, the solvable Lie groups acting simply transitively on the real and complex hyperbolic spaces, $\mathbb{R} H^{4}$ and $\mathbb{C} H^{2}$, respectively, and the semidirect product $\mathbb{C} \rtimes \mathbb{C}$. We show that the spaces $\mathbb{C} H^{2}$ and $\mathbb{C} \rtimes \mathbb{C}$ possess an $\mathbb{R} P^{2}$ of (inequivalent) invariant hypercomplex structures while the remaining groups have only one, up to equivalence. Finally, the corresponding hyperhermitian 4-manifolds are determined.
\end{abstract}

\section{INTRODUCTION}

A hypercomplex structure on a manifold $M$ is a pair $\left\{J_{\alpha}\right\}_{\alpha=1,2}$ of anticommuting complex structures on $M$. A hypercomplex structure on a Lie group $G$ is said to be invariant if left translations by elements of $G$ are holomorphic with respect to both $J_{1}$ and $J_{2}$.

Given $\mathfrak{g}$ a real Lie algebra, a hypercomplex structure (abbreviated $h c s$ ) on $\mathfrak{g}$ is a family $\left\{J_{\alpha}\right\}_{\alpha=1,2}$ of endomorphisms of $\mathfrak{g}$ satisfying the conditions:

$$
\begin{gathered}
J_{\alpha}^{2}=-I, \quad \alpha=1,2, \quad J_{1} J_{2}=-J_{2} J_{1}, \\
N_{\alpha}=0, \quad \alpha=1,2,
\end{gathered}
$$

where $I$ is the identity and $N_{\alpha}$ is the Nijenhuis tensor corresponding to $J_{\alpha}$ :

$$
N_{\alpha}(X, Y)=\left[J_{\alpha} X, J_{\alpha} Y\right]-J_{\alpha}\left(\left[X, J_{\alpha} Y\right]+\left[J_{\alpha} X, Y\right]\right)-[X, Y]
$$

for all $X, Y \in \mathfrak{g}$. Clearly, if $G$ is a Lie group with Lie algebra $\mathfrak{g}$, a hcs on $\mathfrak{g}$ induces by left translations an invariant hypercomplex structure on $G$.

It follows from (1.1) that $J_{1}, J_{2}$ and $J_{3}=J_{1} J_{2}$ give $\mathfrak{g}$ a structure of $\mathbb{H}$-module where $\mathbb{H}$ denotes the quaternions so $\operatorname{dim} \mathfrak{g}=4 n, n \in \mathbb{N}$. Moreover, one verifies that (1.1) and (1.2) imply $N_{3}=0$.

Two hypercomplex structures $\left\{J_{\alpha}\right\}_{\alpha=1,2}$ and $\left\{J_{\alpha}^{\prime}\right\}_{\alpha=1,2}$ on $\mathfrak{g}$ are said to be equivalent if there exists an automorphism $\phi$ of $\mathfrak{g}$ such that $\phi J_{\alpha}=J_{\alpha}^{\prime} \phi$ for $\alpha=1,2$.

Received by the editors March 9, 1994 and, in revised form, September 5, 1995.

1991 Mathematics Subject Classification. Primary 32M10, 53C25, 53C56; Secondary 32M15, $53 \mathrm{C} 55$.

Key words and phrases. Hypercomplex structure (hcs), hyperhermitian metric.

The author was partially supported by Conicor and Conicet (Argentina).

(C)1997 American Mathematical Society 
The existence of invariant hypercomplex structures on compact Lie groups has been studied by Joyce in [4]. Also Spindel et al. consider in [10] products of simple Lie groups and $S^{1}$ factors, studying the existence of hypercomplex structures invariant with respect to a (not necessarily positive definite) metric.

It is the aim of this work to parametrize the equivalence classes of invariant hypercomplex structures on 4-dimensional simply connected real Lie groups (see Section 3) and to determine all the (left invariant) associated hyperhermitian manifolds (see Section 4).

The author would like to thank Prof. I. Dotti Miatello for supervision and invaluable comments, Prof. D. Alekseevsky for suggesting the problem and the referee for drawing [5] to her attention. This work is part of the author's doctoral thesis under the guidance of Prof. I. Dotti Miatello.

\section{Preliminaries}

A $h c s\left\{J_{\alpha}\right\}_{\alpha=1,2}$ on $\mathfrak{g}$ gives a two-sphere $S^{2}$ of complex structures

$$
J_{x}=\sum_{\beta=1}^{3} x_{\beta} J_{\beta}, \quad x_{1}^{2}+x_{2}^{2}+x_{3}^{2}=1,
$$

where $J_{3}=J_{1} J_{2}$. Moreover, every pair of orthogonal vectors $x=\left(x_{1}, x_{2}, x_{3}\right), \quad y=$ $\left(y_{1}, y_{2}, y_{3}\right)$ on the unit sphere $S^{2}$, defines a hypercomplex structure $\left\{J_{x}, J_{y}\right\}$ on $\mathfrak{g}$.

We now prove three lemmas which will be useful in the sequel.

Lemma 2.1. If $\left\{J_{\alpha}\right\}_{\alpha=1,2}$ is a family of endomorphisms of $\mathbb{R}^{4}$ satisfying (1.1) and $W \subset \mathbb{R}^{4}$ is an arbitrary two-dimensional subspace then there exists $x=$ $\left(x_{1}, x_{2}, x_{3}\right) \in S^{2}$ such that $J_{x}=\sum_{\alpha=1}^{3} x_{\alpha} J_{\alpha}$ preserves $W$ (as usual $\left.J_{3}=J_{1} J_{2}\right)$.

Proof. We take a basis $\{X, Y\}$ of $W$ and we write $Y=x_{0} X+\sum_{\alpha=1}^{3} x_{\alpha} J_{\alpha} X$. We may assume that $x_{1}^{2}+x_{2}^{2}+x_{3}^{2}=1$, and now the lemma follows by taking $x=\left(x_{1}, x_{2}, x_{3}\right)$.

Lemma 2.2. If $J_{\alpha}$ is an endomorphism of $\mathfrak{g}$ such that $J_{\alpha}^{2}=-I$ and $\left\{X_{1}, J_{\alpha} X_{1}\right.$, $\left.\ldots, X_{n}, J_{\alpha} X_{n}\right\}$ is a basis of $\mathfrak{g}$, then $N_{\alpha} \equiv 0$ if and only if $N_{\alpha}\left(X_{i}, X_{j}\right)=0, \forall i<j$.

Proof. It is easy to see that $N_{\alpha}\left(J_{\alpha} X, Y\right)=-J_{\alpha} N_{\alpha}(X, Y)$. The lemma now follows by using that $N_{\alpha}$ is skew-symmetric.

Lemma 2.3. If $\left\{J_{\alpha}\right\}_{\alpha=1,2}$ is a family of endomorphisms of a real vector space $V$ satisfying (1.1) then $V$ admits an inner product such that $J_{1}$ and $J_{2}$ are orthogonal. If $\operatorname{dim} V=4$ then such an inner product is unique up to a positive multiple.

Proof. Let $\langle$,$\rangle be an arbitrary inner product and define:$

$$
(X, Y)=\langle X, Y\rangle+\sum_{\alpha=1}^{3}\left\langle J_{\alpha} X, J_{\alpha} Y\right\rangle, \quad \forall X, Y \in V,
$$

where $J_{3}=J_{1} J_{2}$. It is clear that (, ) is an inner product with the desired properties. If $\operatorname{dim} V=4$ and $(,)_{1}$ is an inner product on $V$ such that $J_{1}$ and $J_{2}$ are orthogonal, let $T \in \operatorname{End}(V)$ be such that $(X, Y)_{1}=(T X, Y) \quad \forall X, Y \in V$. If $V_{\lambda} \quad(\lambda>0)$ is the eigenspace of $T$ corresponding to the eigenvalue $\lambda$, then $J_{\alpha}$ preserves $V_{\lambda}$ $(\alpha=1,2) ;$ thus $\operatorname{dim} V_{\lambda} \equiv 0(\bmod 4)$. Hence $V_{\lambda}=V$ and $(,)_{1}=\lambda($,$) .$ 


\section{Classification of hes}

In this section we shall obtain the main results of this paper. We will parametrize the equivalence classes of hcs on each four-dimensional real Lie algebra (which does admit one). From now on $\mathfrak{g}$ will be a 4 -dimensional real Lie algebra with center $\mathfrak{z}$ and derived Lie algebra $\mathfrak{g}^{\prime}=[\mathfrak{g}, \mathfrak{g}]$. We will consider separately the cases $\mathfrak{g}$ nonsolvable and $\mathfrak{g}$ solvable.

We first prove:

Theorem 3.1. If $\mathfrak{g}$ is not solvable then $\mathfrak{g}$ admits a hcs if and only if $\mathfrak{g} \cong \mathbb{R} \oplus \mathfrak{s o}(3)$. Moreover, the hcs on $\mathbb{R} \oplus \mathfrak{s o}(3)$ is unique up to equivalence.

Proof. The if part of the first assertion follows by exhibiting a hcs $\left\{J_{\alpha}\right\}_{\alpha=1,2}$ on $\mathbb{R} \oplus \mathfrak{s o}(3)$. Let $\{Z, X, Y, W\}$ be a basis of $\mathbb{R} \oplus \mathfrak{s o}(3)$ such that $Z \in \mathbb{R}$ and

$$
[X, Y]=W, \quad[Y, W]=X, \quad[W, X]=Y .
$$

Let $J_{1}, J_{2} \in \operatorname{End}(\mathbb{R} \oplus \mathfrak{s o}(3))$ be defined by

$$
\begin{array}{lll}
J_{1} Z=X, & J_{1} Y=W, & J_{1}^{2}=-I, \\
J_{2} Z=Y, & J_{2} W=X, & J_{2}^{2}=-I .
\end{array}
$$

It is clear that $J_{1}, J_{2}$ anticommute. Furthermore

$$
\begin{aligned}
N_{1}(Z, Y) & =\left[J_{1} Z, J_{1} Y\right]-J_{1}\left[Z, J_{1} Y\right]-J_{1}\left[J_{1} Z, Y\right]-[Z, Y] \\
& =[X, W]-J_{1}[X, Y]=0
\end{aligned}
$$

and by Lemma $2.2, J_{1}$ is integrable. A similar computation shows that $N_{2}(Z, W)$ vanishes so $\mathcal{H}=\left\{J_{\alpha}\right\}_{\alpha=1,2}$ defines a hcs on $\mathbb{R} \oplus \mathfrak{s o}(3)$.

We assume now that $\mathfrak{g}$ admits a $h c s\left\{J_{\alpha}\right\}_{\alpha=1,2}$. Since $\mathfrak{g}$ is not solvable then, by using the Levi decomposition of $\mathfrak{g}$ and the classification of real simple 3 -dimensional Lie algebras, we get that $\mathfrak{g} \cong \mathfrak{z} \oplus \mathfrak{s o}(3)$ or $\mathfrak{g} \cong \mathfrak{z} \oplus \mathfrak{s l}(2, \mathbb{R})$, a direct sum of ideals. If $Z$ is a nonzero element in $\mathfrak{z}$ we define

$$
X=J_{1} Z, \quad Y=J_{2} Z, \quad W=J_{1} J_{2} Z .
$$

Then $\{Z, X, Y, W\}$ is a basis of $\mathfrak{g}$. We compute $[X, Y],[Y, W],[W, X]$. Write $[X, Y]=a Z+b X+c Y+d W$. Since $N_{1}(Z, Y)=0=N_{2}(Z, X)$ we have:

$$
J_{1}[X, Y]=[X, W], \quad J_{2}[X, Y]=[Y, W]
$$

Thus

$$
[W, X]=b Z-a X+d Y-c W, \quad[Y, W]=-c Z+d X+a Y-b W .
$$

Now, the coefficient of $Z$ in $[[X, Y], W]+[[Y, W], X]+[[W, X], Y]$ is $a^{2}+b^{2}+c^{2}$; hence $a=b=c=0$ and

$$
[X, Y]=d W, \quad[Y, W]=d X, \quad[W, X]=d Y
$$

Since $d \neq 0(\mathfrak{g}$ is not abelian) then $\{X, Y, W\}$ generates a three dimensional Lie algebra isomorphic to $\mathfrak{s o}(3)$.

If $\left\{J_{\alpha}^{\prime}\right\}_{\alpha=1,2}$ is another $h c s$ on $\mathfrak{g}$, setting $J_{3}^{\prime}=J_{1}^{\prime} J_{2}^{\prime}$, it follows from the above procedure that there exists $d^{\prime} \neq 0$ such that $\left[J_{\alpha}^{\prime} Z, J_{\beta}^{\prime} Z\right]=d^{\prime} J_{\gamma}^{\prime} Z$, where $(\alpha, \beta, \gamma)$ is a cyclic permutation of $(1,2,3)$ (this is analogous to $(3.1)$ ). Let $\phi \in \operatorname{End}(\mathfrak{g})$ be defined by $\phi Z=\frac{d}{d^{\prime}} Z$ and $\phi J_{\alpha}=J_{\alpha}^{\prime} \phi$, for $\alpha=1,2$. One verifies that $\phi$ is an automorphism of $\mathfrak{g}$; hence $\left\{J_{\alpha}\right\}_{\alpha=1,2}$ and $\left\{J_{\alpha}^{\prime}\right\}_{\alpha=1,2}$ are equivalent. This concludes the proof of the theorem. 
Remark 3.1. The simply connected Lie group with Lie algebra $\mathbb{R} \oplus \mathfrak{s o}(3)$ is the multiplicative group $\mathbb{H}^{*}$ of nonzero quaternions. In the next section we show that the associated hyperhermitian metric corresponds to the riemannian product $\mathbb{R} \times S^{3}$ with the canonical metrics.

Remark 3.2. We have actually proved in the theorem that if $\mathfrak{z} \neq\{0\}$ then $J_{1 \mathfrak{z}} \oplus$ $J_{2} \mathfrak{z} \oplus J_{3 \mathfrak{z}}$ is either isomorphic to $\mathfrak{s o}(3)$ or is abelian. Thus a 4 -dimensional Lie algebra $\mathfrak{g}$ with nontrivial center admits a hcs if and only if $\mathfrak{g} \cong \mathbb{R} \oplus \mathfrak{s o}(3)$ or $\mathfrak{g}$ is abelian.

Remark 3.3. According to Theorem $3.1, \mathbb{R} \oplus \mathfrak{s l}(2, \mathbb{R})$ does not admit a hcs. On the other hand, it does admit an invariant complex structure by results of D. Snow [8].

Remark 3.4. As a consequence of Theorem 3.1 we also obtain that quotients of $\mathbb{R} \times S^{3}$ by discrete central subgroups also admit invariant $h c s: \mathbb{R} \times S O(3), S^{1} \times$ $S^{3}$ and $S^{1} \times S O(3)$. The last two spaces are Hopf surfaces that appear in Boyer's classification of compact hyperhermitian 4-manifolds (cf. [1]). Note that via the diffeomorphism $S^{1} \times S^{3} \cong U(2)$ we can give $U(2)$ a hypercomplex structure (which is not necessarily invariant).

In the case when $\mathfrak{g}$ is solvable we will analyze separately the cases $\operatorname{dim} \mathfrak{g}^{\prime}=$ $0,1,2,3$.

If $\operatorname{dim} \mathfrak{g}^{\prime}=0$ then $\mathfrak{g}$ is abelian. A hcs on $\mathfrak{g}$ can be obtained by choosing two endomorphisms satisfying condition (1.1). In this situation the integrability condition (1.2) is automatically satisfied and one can show, with similar arguments to those in the complex case $([6])$, that there is a one to one correspondence between hypercomplex structures on $\mathfrak{g}$ and points in the space $G L(4 n, \mathbb{R}) / G L(n, \mathbb{H})$. The correspondence is established by fixing a $h c s\left\{J_{\alpha}^{0}\right\}_{\alpha=1,2}$ and sending $G L(4 n, \mathbb{R}) \ni$ $T \rightarrow\left\{T J_{\alpha}^{0} T^{-1}\right\}_{\alpha=1,2}$. In particular, it follows that every hcs is equivalent to $\left\{J_{\alpha}^{0}\right\}_{\alpha=1,2}$.

If the dimension of $\mathfrak{g}^{\prime}$ is 1 we have

Proposition 3.2. If $\operatorname{dim} \mathfrak{g}^{\prime}=1$ then $\mathfrak{g}$ does not admit any hcs.

Proof. If $\mathfrak{g}$ admits a hcs we may assume, in view of Remark 3.2 , that $\mathfrak{z}=\{0\}$. Let $X$ be a nonzero element of $\mathfrak{g}^{\prime}$. There exists $Y \in \mathfrak{g}$ such that $[Y, X]=X$. Then $\mathfrak{g}$ decomposes

$$
\mathfrak{g}=\operatorname{ker}\left(a d_{X}\right) \cap \operatorname{ker}\left(a d_{Y}\right) \oplus \mathbb{R} X \oplus \mathbb{R} Y .
$$

By applying the Jacobi identity to $U, V, Y$, where $U, V \in \operatorname{ker}\left(a d_{X}\right) \cap \operatorname{ker}\left(a d_{Y}\right)$, we get that $[U, V]=0$; hence $\mathfrak{z}=\operatorname{ker}\left(a d_{X}\right) \cap \operatorname{ker}\left(a d_{Y}\right)$, a contradiction.

Let $A f f(\mathbb{C})$ be the affine motion group of $\mathbb{C}$ under composition. It is isomorphic to the semidirect product of $\mathbb{C}$ with $\mathbb{C}^{*}$. Its Lie algebra $\mathfrak{a f f}(\mathbb{C})$ decomposes as a direct sum of an abelian ideal (which corresponds to the normal subgroup of translations) plus an abelian subalgebra (corresponding to multiplication by a scalar in $\mathbb{C}^{*}$ ). Moreover, there are bases $X, Y$ of the ideal and $Z, W$ of the subalgebra such that the following relations hold:

$$
\begin{aligned}
{[X, Z] } & =X, & {[Y, Z] } & =Y, \\
{[X, W] } & =Y, & {[Y, W] } & =-X .
\end{aligned}
$$

The next theorem settles the case $\operatorname{dim} \mathfrak{g}^{\prime}=2$. 
Theorem 3.3. If $\operatorname{dim} \mathfrak{g}^{\prime}=2$ then

(i) $\mathfrak{g}$ admits a hcs if and only if $\mathfrak{g} \cong \mathfrak{a f f}(\mathbb{C})$;

(ii) the equivalence classes of hcs on $\mathfrak{g}$ are parametrized by the space $\mathbb{R} P^{2}$.

Proof. (i) We show first that $\mathfrak{a f f}(\mathbb{C})$ admits a hcs. Let $\mathcal{H}=\left\{J_{\alpha}\right\}_{\alpha=1,2}$ be the following family of endomorphisms of $\mathfrak{a f f}(\mathbb{C})$ :

$$
\begin{array}{lll}
J_{1} X=-W, & J_{1} Y=Z, & J_{1}^{2}=-I, \\
J_{2} X=Y, & J_{2} Z=-W, & J_{2}^{2}=-I .
\end{array}
$$

It is easy to check the integrability of $J_{1}$ and $J_{2}$ by using Lemma 2.2 .

Conversely, assume now that $\left\{J_{\alpha}\right\}_{\alpha=1,2}$ defines a hcs on $\mathfrak{g}$. Changing $\left\{J_{\alpha}\right\}_{\alpha=1,2}$, if necessary (see (2.1) and Lemma 2.1), we may assume that $J_{2}: \mathfrak{g}^{\prime} \rightarrow \mathfrak{g}^{\prime}$; hence $\mathfrak{g}=\mathfrak{g}^{\prime} \oplus J_{1} \mathfrak{g}^{\prime}$. Let $\left\{X^{\prime}, Y^{\prime}\right\}$ be a basis of $\mathfrak{g}^{\prime}$ where $Y^{\prime}=J_{2} X^{\prime}$. It follows that $\left\{X^{\prime}, Y^{\prime}, J_{1} X^{\prime}, J_{1} Y^{\prime}\right\}$ is a basis of $\mathfrak{g}$. There exist two skew-symmetric, bilinear forms $\alpha, \beta$ on $\mathfrak{g}$ such that

$$
[V, W]=\alpha(V, W) X^{\prime}+\beta(V, W) Y^{\prime} \quad \forall V, W \in \mathfrak{g} .
$$

The integrability condition $N_{1}\left(X^{\prime}, Y^{\prime}\right)=0$ and the fact that $\mathfrak{g}^{\prime}$ is abelian (since $\mathfrak{g}$ is solvable) yield

$$
\left[J_{1} X^{\prime}, J_{1} Y^{\prime}\right]=0, \quad\left[X^{\prime}, J_{1} Y^{\prime}\right]=\left[Y^{\prime}, J_{1} X^{\prime}\right]
$$

and from $N_{2}\left(X^{\prime}, J_{1} X^{\prime}\right)=0$ we obtain

$$
\left[X^{\prime}, J_{1} X^{\prime}\right]=-\left[Y^{\prime}, J_{1} Y^{\prime}\right]
$$

The Jacobi identity gives

$$
\alpha\left(X^{\prime}, J_{1} X^{\prime}\right)=\beta\left(X^{\prime}, J_{1} Y^{\prime}\right), \quad \alpha\left(X^{\prime}, J_{1} Y^{\prime}\right)=-\beta\left(X^{\prime}, J_{1} X^{\prime}\right),
$$

and therefore the bracket in $\mathfrak{g}$, determined by $c=\alpha\left(X^{\prime}, J_{1} X^{\prime}\right)$ and $d=\alpha\left(X^{\prime}, J_{1} Y^{\prime}\right)$, looks as follows:

$$
\begin{array}{llrl}
{\left[X^{\prime}, J_{1} X^{\prime}\right]} & =c X^{\prime}-d Y^{\prime}, & {\left[Y^{\prime}, J_{1} X^{\prime}\right]} & =d X^{\prime}+c Y^{\prime}, \\
{\left[X^{\prime}, J_{1} Y^{\prime}\right]} & =d X^{\prime}+c Y^{\prime}, & {\left[Y^{\prime}, J_{1} Y^{\prime}\right]} & =-c X^{\prime}+d Y^{\prime},
\end{array}
$$

and we must have $c \neq 0$ or $d \neq 0$ because $\operatorname{dim} \mathfrak{g}^{\prime}=2$. Taking

$$
\begin{gathered}
X=\left(c^{2}+d^{2}\right)^{-1}\left(d X^{\prime}+c Y^{\prime}\right), \quad Y=\left(c^{2}+d^{2}\right)^{-1}\left(-c X^{\prime}+d Y^{\prime}\right), \\
Z=\left(c^{2}+d^{2}\right)^{-1}\left(c J_{1} X^{\prime}+d J_{1} Y^{\prime}\right), \quad W=\left(c^{2}+d^{2}\right)^{-1}\left(-d J_{1} X^{\prime}+c J_{1} Y^{\prime}\right),
\end{gathered}
$$

it is easy to see that

$$
\begin{aligned}
{[X, Z] } & =X, & {[Y, Z] } & =Y, \\
{[X, W] } & =Y, & {[Y, W] } & =-X,
\end{aligned}
$$

so that $\mathfrak{g} \cong \mathfrak{a f f}(\mathbb{C})$, as asserted.

We note that $J_{1}$ and $J_{2}$ take the following form relative to the basis $\{X, Y, Z, W\}$ :

$$
\begin{aligned}
& J_{1} X=a Z-b W, \\
& J_{1} Y=b Z+a W, \\
& J_{1}^{2}=-I, \\
& J_{2} X=Y \text {, } \\
& J_{2} Z=-W \text {, } \\
& J_{2}^{2}=-I \text {, }
\end{aligned}
$$

where $a=2 c d\left(c^{2}+d^{2}\right)^{-1}$ and $b=\left(d^{2}-c^{2}\right)\left(c^{2}+d^{2}\right)^{-1}$; hence $a^{2}+b^{2}=1$. Let $\left\{J_{\alpha}^{0}\right\}_{\alpha=1,2}$ be the hcs obtained by setting

$$
J_{1}^{0} X=-W, \quad J_{1}^{0} Y=Z, \quad\left(J_{1}^{0}\right)^{2}=-I,
$$


and $J_{2}^{0}=J_{2}$. We claim that $\left\{J_{\alpha}\right\}_{\alpha=1,2}$ and $\left\{J_{\alpha}^{0}\right\}_{\alpha=1,2}$ are equivalent. In fact, one shows that $\phi \in \operatorname{End}(\mathfrak{g})$ defined by $\phi X=b X+a Y, \phi Y=-a X+b Y, \phi Z=$ $Z$ and $\phi W=W$ gives an automorphism of $\mathfrak{g}$ such that $\phi J_{\alpha}=J_{\alpha}^{0} \phi, \alpha=1,2$.

(ii) We have actually proved in (i) that all hypercomplex structures $\left\{J_{\alpha}\right\}_{\alpha=1,2}$ with the property that $J_{2}$ preserves $\mathfrak{g}^{\prime}$ are equivalent. Given a fixed structure $\left\{J_{\alpha}^{0}\right\}_{\alpha=1,2}$ having this property, we define $S O(3)^{0}=\left\{\left\{J_{x}^{0}, J_{y}^{0}\right\}: x, y \in S^{2}, x \perp y\right\}$ where $J_{x}^{0}=\sum_{\alpha=1}^{3} x_{\alpha} J_{\alpha}^{0}, J_{y}^{0}=\sum_{\alpha=1}^{3} y_{\alpha} J_{\alpha}^{0}$ with $J_{3}^{0}=J_{1}^{0} J_{2}^{0}$. Then any $h c s$ on $\mathfrak{g}=\mathfrak{a f f}(\mathbb{C})$ is equivalent to one lying in $S O(3)^{0}$. To prove this we take an arbitrary $h c s\left\{J_{\alpha}\right\}_{\alpha=1,2}$. By Lemma 2.1 there exists $q \in S^{2}$ such that $J_{q}=\sum_{\alpha=1}^{3} q_{\alpha} J_{\alpha}$ preserves $\mathfrak{g}^{\prime}$, where $J_{3}=J_{1} J_{2}$. If $p \in S^{2}$ is orthogonal to $q$ then $\left\{J_{p}, J_{q}\right\}$ is a $h c s$ equivalent to $\left\{J_{\alpha}^{0}\right\}_{\alpha=1,2}$, say by an automorphism $\phi$. Let $x, y$ be the the first two rows of $A^{-1}$, where $A$ is the matrix with rows $p, q, p \wedge q$. Then $\phi$ is also an equivalence between $\left\{J_{\alpha}\right\}_{\alpha=1,2}$ and $\left\{J_{x}^{0}, J_{y}^{0}\right\}$.

We have proved that the map $(x, y, x \wedge y) \rightarrow\left\{J_{x}^{0}, J_{y}^{0}\right\}$ is surjective from $S O(3)$ onto the set of equivalence classes of $h c s$ on $\mathfrak{g}$. Assume that $\left\{J_{\alpha}^{0}\right\}_{\alpha=1,2}$ is the structure defined in (i); then one shows that $\left\{J_{x}^{0}, J_{y}^{0}\right\}$ and $\left\{J_{p}^{0}, J_{q}^{0}\right\}$ are equivalent if and only if there exists $A=\left(a_{i j}\right)_{1 \leq i, j \leq 2} \in O(2)$ such that $x=\tilde{A} p, y=\tilde{A} q$, where $\tilde{A}=\left(\begin{array}{ccc}a_{22} & 0 & a_{12} \\ 0 & \operatorname{det} A & 0 \\ a_{21} & 0 & a_{11}\end{array}\right)$. Therefore, the equivalence classes of hcs on $\mathfrak{g}$ are in one to one correspondence with points in the space $O(2) \backslash S O(3)=\mathbb{R} P^{2}$.

Remark 3.5. Snow classified in [9] the complex structures on a 4-dimensional solvable real Lie algebra $\mathfrak{g}$ such that $\operatorname{dim} \mathfrak{g}^{\prime} \leq 2$. The Lie algebra appearing in Theorem 3.3 was denoted by $S 11$ in [9], where it is shown that $S 11$ admits four distinct families of equivalence classes of complex structures. The structures $J_{1}^{0}$ and $J_{2}^{0}$ of Theorem 3.3 represent nonequivalent complex structures belonging to different families.

To settle the classification we consider now the case $\operatorname{dim} \mathfrak{g}^{\prime}=3$. We first introduce two families of solvable Lie algebras which are related to the real and complex hyperbolic spaces $\mathbb{R} H^{n}$ and $\mathbb{C} H^{n}$, respectively. It is well known that either space admits a solvable group of isometries acting simply transitively on it (cf. [3]), thus it is isometric to a (simply connected) solvable Lie group with a left invariant metric. Let $(\mathfrak{s},\langle\rangle$,$) denote the corresponding solvable Lie algebra with inner product$ $\langle$,$\rangle . Then ([3]) \mathfrak{s}$ has an orthogonal decomposition $\mathfrak{s}=\mathbb{R} A \oplus \mathfrak{a}_{1} \oplus \mathfrak{a}_{2}$ such that $\langle A, A\rangle=1, \mathfrak{s}^{\prime}=\mathfrak{a}_{1} \oplus \mathfrak{a}_{2},\left[\mathfrak{s}^{\prime}, \mathfrak{s}^{\prime}\right]=\mathfrak{a}_{2}$ and $\left[\mathfrak{s}^{\prime}, \mathfrak{a}_{2}\right]=0$, where $\operatorname{dim} \mathfrak{a}_{1}=s\left(\operatorname{dim} \mathfrak{a}_{2}+1\right)$ for some positive integer $s$. $A$ acts by the identity on $\mathfrak{a}_{2}$ and by one half the identity on $\mathfrak{a}_{1}$.

When $\mathfrak{a}_{2}=0$ then $\mathfrak{a}_{1}$ is abelian and the $n$-dimensional simply connected Lie group with left invariant metric associated to $(\mathfrak{s},\langle\rangle$,$) is isometric to \mathbb{R} H^{n}$ (cf. [3]).

If $\mathfrak{a}_{2}=\mathbb{R} X(\langle X, X\rangle=1)$ and $\mathfrak{a}_{1}$ has an orthonormal basis $\left\{Y_{1}, Z_{1}, \ldots, Y_{n-1}, Z_{n-1}\right\}$ such that $\left[Z_{i}, Y_{j}\right]=-\left[Y_{j}, Z_{i}\right]=\delta_{i j} X$, then the corresponding $2 n$-dimensional simply connected Lie group with left invariant metric is isometric to $\mathbb{C} H^{n}$ (cf. [3]).

Theorem 3.4. If $\operatorname{dim} \mathfrak{g}^{\prime}=3$ and $\mathfrak{g}$ is solvable then one of the following holds:

(a) $\mathfrak{g}^{\prime}$ is abelian or $(\mathbf{b}) \mathfrak{g}^{\prime}$ is a Heisenberg algebra.

In case $(\mathbf{a}) \mathfrak{g}$ admits a hcs if and only if $\mathfrak{g}$ corresponds to the space $\mathbb{R} H^{4}$. Moreover, $\mathfrak{g}$ has a unique hcs, up to equivalence. 
In case $(\mathbf{b}) \mathfrak{g}$ admits a hcs if and only if it corresponds to the space $\mathbb{C} H^{2}$. The equivalence classes of hcs on $\mathfrak{g}$ are parametrized by the space $\mathbb{R} P^{2}$.

Proof. Since $\mathfrak{g}^{\prime}$ is nilpotent, the first assertion in the theorem follows since the only three-dimensional nilpotent, nonabelian Lie algebra is the Heisenberg algebra.

We assume now that condition (a) holds. If $\mathfrak{g}$ admits a $h c s\left\{J_{\alpha}\right\}_{\alpha=1,2}$, let $\langle$,$\rangle be$ an inner product such that $J_{\alpha}$ is orthogonal for $\alpha=1,2,3$ with $J_{3}=J_{1} J_{2}$ (Lemma 2.3). We fix a nonzero element $A \in \mathfrak{g}$ orthogonal to $\mathfrak{g}^{\prime}$ with respect to $\langle$,$\rangle . Since$ clearly $\left\{J_{\alpha} A\right\}_{\alpha=1,2,3}$ is a basis of $\mathfrak{g}^{\prime}$, we must have

$$
\left[J_{\alpha} A, J_{\beta} A\right]=0 \quad \forall \alpha, \beta .
$$

If $(\alpha, \beta, \gamma)$ is a cyclic permutation of the indices $(1,2,3)$, by the integrability of $J_{\alpha}$ we obtain

$$
J_{\alpha}\left[A, J_{\beta} A\right]=\left[A, J_{\alpha} J_{\beta} A\right], \quad J_{\alpha}\left[A, J_{\gamma} A\right]=\left[A, J_{\alpha} J_{\gamma} A\right] .
$$

If we now compute $a d_{A}: \mathfrak{g}^{\prime} \rightarrow \mathfrak{g}^{\prime}$ with respect to the basis $\left\{J_{\alpha} A\right\}_{\alpha=1,2,3}$ and use (3.2) we obtain

$$
a d_{A \mid \mathfrak{g}^{\prime}}=\lambda I
$$

which shows that $\mathfrak{g}$ is isomorphic to the solvable Lie algebra corresponding to the real hyperbolic space. Conversely, given this Lie algebra, we now exhibit a hcs on it. We choose a basis $\{X, Y, Z\}$ of $\mathfrak{g}^{\prime}$ and if $A \notin \mathfrak{g}^{\prime}$ satisfies (3.3), define $\mathcal{H}=\left\{J_{\alpha}\right\}_{\alpha=1,2}$ as follows

$$
\begin{array}{lll}
J_{1} A=X, & J_{1} Y=Z, & J_{1}^{2}=-I, \\
J_{2} A=Y, & J_{2} Z=X, & J_{2}^{2}=-I .
\end{array}
$$

It is easy to check that $N_{1}(A, Y)=0$ and $N_{2}(A, Z)=0$, so in view of Lemma $2.2 J_{1}$ and $J_{2}$ are integrable. Furthermore, one obtains that all hypercomplex structures on $\mathfrak{g}$ are equivalent to $\mathcal{H}$. In fact, given another $h c s\left\{J_{\alpha}^{\prime}\right\}_{\alpha=1,2}$ with corresponding inner product $\langle,\rangle^{\prime}$, we take $A^{\prime} \in \mathfrak{g}$ orthogonal to $\mathfrak{g}^{\prime}$ with respect to $\langle,\rangle^{\prime}$ and in the same way as above we obtain $a d_{A^{\prime} \mid \mathfrak{g}^{\prime}}=\lambda^{\prime} I$ for some $\lambda^{\prime} \neq 0$. Let $\phi \in \operatorname{End}(\mathfrak{g})$ be defined by $\phi A=\frac{\lambda}{\lambda^{\prime}} A^{\prime}$ and $\phi J_{\alpha}=J_{\alpha}^{\prime} \phi$, for $\alpha=1,2$. One verifies that $\phi$ is an automorphism of $\mathfrak{g}$; hence it sets the desired equivalence.

Assume now that (b) holds. If $\mathfrak{g}$ is the Lie algebra that corresponds to $\mathbb{C} H^{2}$ then it has a basis $\{A, X, Y, Z\}$ such that

$$
\begin{aligned}
{[A, X] } & =X, & {[Z, Y] } & =X, \\
{[A, Y] } & =\frac{1}{2} Y, & {[A, Z] } & =\frac{1}{2} Z .
\end{aligned}
$$

We define $J_{1}, J_{2} \in \operatorname{End}(\mathfrak{g})$ by the following conditions:

$$
\begin{aligned}
& J_{1} A=X, \quad J_{1} Y=Z, \quad J_{1}^{2}=-I, \\
& J_{2} A=\frac{\sqrt{2}}{2} Z, \quad J_{2} X=\frac{\sqrt{2}}{2} Y, \quad J_{2}^{2}=-I .
\end{aligned}
$$

The integrability of $J_{1}$ and $J_{2}$ is easily verified by direct calculation.

Conversely, if $\mathfrak{g}$ satisfies (b) and admits a $h c s\left\{J_{\alpha}\right\}_{\alpha=1,2}$, let $\langle$,$\rangle be an inner$ product such that $J_{\alpha}$ is orthogonal for $\alpha=1,2$ (cf. Lemma 2.3). We choose a nonzero element $\tilde{A}$ in the orthogonal complement of $\mathfrak{g}^{\prime}$ and by Lemma 2.1 we may assume that $J_{1} \tilde{A} \in \mathfrak{z}\left(\mathfrak{g}^{\prime}\right)$. We consider the following basis of $\mathfrak{g}: \tilde{A}, \tilde{X}=J_{1} \tilde{A}, Z=$ $J_{2} \tilde{A}, Y=J_{2} \tilde{X}$ (note that $\{\tilde{X}, Z, Y\}$ is a basis of $\mathfrak{g}^{\prime}$ ). Since $\tilde{X} \in \mathfrak{z}\left(\mathfrak{g}^{\prime}\right)$ it is easy 
to verify that $\mathbb{R} \tilde{X}$ is an ideal of $\mathfrak{g}$, so there exists $\lambda \in \mathbb{R}$ such that $[\tilde{A}, \tilde{X}]=\lambda \tilde{X}$. By Remark $3.2 \mathfrak{z}=\{0\}$; hence $\lambda \neq 0$. By assumption $\mathfrak{g}^{\prime}$ is a Heisenberg algebra with center $\mathbb{R} \tilde{X}$ so there exists $\alpha \in \mathbb{R}(\alpha \neq 0)$ such that $[Z, Y]=\alpha \tilde{X}$. From $N_{2}(\tilde{X}, Z)=0$ and $N_{1}(\tilde{A}, Z)=0$ one obtains

$$
[\tilde{A}, Y]=(\lambda-\alpha) Y, \quad[\tilde{A}, Z]=J_{1}[\tilde{A}, Y]=(\lambda-\alpha) Z .
$$

Now the Jacobi identity applied to $\tilde{A}, Z, Y$ yields $\alpha=\frac{\lambda}{2}$. Taking $A=\frac{1}{\lambda} \tilde{A}, X=$ $\frac{\lambda}{2} \tilde{X}, Z, Y$ and using (3.4), it is straightforward to show that $\mathfrak{g}$ is the Lie algebra corresponding to the complex hyperbolic space. In the above basis $J_{1}$ and $J_{2}$ take the following form:

$$
\begin{aligned}
& J_{1} A=\frac{2}{\lambda^{2}} X, \quad J_{1} Y=Z, \quad J_{1}^{2}=-I, \\
& J_{2} A=\frac{1}{\lambda} Z, \quad J_{2} X=\frac{\lambda}{2} Y, \quad J_{2}^{2}=-I,
\end{aligned}
$$

and if we call $J_{1}^{\prime}$ and $J_{2}^{\prime}$ the structure obtained when we replace $\lambda$ by $\lambda^{\prime}$, it is easy to verify that $\phi \in \operatorname{End}(\mathfrak{g})$ satisfying $\phi A=A$ and $\phi J_{\alpha}=J_{\alpha}^{\prime} \phi$, for $\alpha=1,2$, is an automorphism of $\mathfrak{g}$ that establishes an equivalence between $\left\{J_{\alpha}^{\prime}\right\}_{\alpha=1,2}$ and $\left\{J_{\alpha}\right\}_{\alpha=1,2}$. We have actually proved that all hypercomplex structures $\left\{J_{\alpha}\right\}_{\alpha=1,2}$ with the property that $J_{1}$ leaves $\mathbb{R} A \oplus \mathfrak{z}\left(\mathfrak{g}^{\prime}\right)$ invariant are equivalent. Let us fix $\lambda=\sqrt{2}$ and denote by $\left\{J_{\alpha}^{0}\right\}_{\alpha=1,2}$ the associated hcs. If we define $S O(3)^{0}$ as we did in (ii) of Theorem 3.3 , by similar arguments we get that every hcs on $\mathfrak{g}$ is equivalent to one lying in $S O(3)^{0}$. One shows that $\left\{J_{x}^{0}, J_{y}^{0}\right\}$ and $\left\{J_{p}^{0}, J_{q}^{0}\right\}$ are equivalent if and only if there exists $A \in O(2)$ such that $x=\tilde{A} p, y=\tilde{A} q$, where $\tilde{A}=\left(\operatorname{det}_{A}\right)$. This condition, which is analogous to the one obtained in Theorem 3.3, settles the parametrization.

\section{The ASSOCIATED HYPERHERMITIAN MANIFOLDS}

Let $\left(M,\left\{J_{\alpha}\right\}_{\alpha=1,2}\right)$ be a connected hypercomplex manifold. A metric $\langle$,$\rangle on M$ is said to be hyperhermitian if the endomorphisms $\left\{J_{\alpha}\right\}_{\alpha=1,2}$ are orthogonal with respect to $\langle$,$\rangle . It turns out from Lemma 2.3$ that, if $\operatorname{dim} M=4$, all hyperhermitian metrics (relative to a fixed structure) are conformally equivalent. A differentiable map $f:\left(M,\left\{J_{\alpha}\right\}_{\alpha=1,2}\right) \rightarrow\left(M^{\prime},\left\{J_{\alpha}^{\prime}\right\}_{\alpha=1,2}\right)$ between two hypercomplex manifolds is said to be hypercomplex if its differential $d f$ satisfies $d f J_{\alpha}=J_{\alpha}^{\prime} d f$, for $\alpha=1,2$. Note that the pull-back of a hyperhermitian metric by a hypercomplex immersion is hyperhermitian.

From now on $G$ will denote a simply connected 4-dimensional real Lie group admitting an invariant hypercomplex structure. A left invariant metric on $G$ is said to be invariant hyperhermitian if it is hyperhermitian with respect to some invariant $h c s$ on $G$. In this section we obtain the invariant hyperhermitian metrics on $G$. The following result states that, on a given $G$, all such metrics are equivalent up to homotheties.

Proposition 4.1. If $\langle$,$\rangle and \langle,\rangle^{\prime}$ are invariant hyperhermitian metrics on $G$ then there exists a real number $\lambda>0$ such that $(G, \lambda\langle\rangle$,$) is isometric to \left(G,\langle,\rangle^{\prime}\right)$.

Proof. By assumption there exist invariant hypercomplex structures $\left\{J_{\alpha}\right\}_{\alpha=1,2}$ and $\left\{J_{\alpha}^{\prime}\right\}_{\alpha=1,2}$ on $G$ corresponding to $\langle$,$\rangle and \langle,\rangle^{\prime}$, respectively. Both invariant hypercomplex structures are determined by the endomorphisms they induce on the Lie algebra $\mathfrak{g}$ of $G$. We still denote these endomorphisms by $\left\{J_{\alpha}\right\}_{\alpha=1,2}$ and $\left\{J_{\alpha}^{\prime}\right\}_{\alpha=1,2}$, 
and they are hcs on $\mathfrak{g}$. From the results of Section 3 we know that there exists a $h c s\left\{J_{\alpha}^{0}\right\}_{\alpha=1,2}$ on $\mathfrak{g}$ such that any other $h c s$ is equivalent to one which lies in the corresponding space $S O(3)^{0}$. Without loss of generality we may assume that one of the structures, for instance $\left\{J_{\alpha}^{\prime}\right\}_{\alpha=1,2}$, is $\left\{J_{\alpha}^{0}\right\}_{\alpha=1,2}$.

Let $x, y \in S^{2}, x \perp y$ and $\phi \in A u t(\mathfrak{g})$ such that $\left\{J_{\alpha}\right\}_{\alpha=1,2}$ is equivalent to $\left\{J_{x}^{0}, J_{y}^{0}\right\}$ via $\phi$. Let $\tilde{\phi}$ be the automorphism of $G$ induced by $\phi$ and let $\langle,\rangle^{x y}$ be a left invariant hyperhermitian metric with respect to $\left\{J_{x}^{0}, J_{y}^{0}\right\}$. The automorphism $\tilde{\phi}$ : $\left(G,\left\{J_{\alpha}\right\}_{\alpha=1,2}\right) \rightarrow\left(G,\left\{J_{x}^{0}, J_{y}^{0}\right\}\right)$ is hypercomplex; then $\tilde{\phi}^{*}\langle,\rangle^{x y}$ is hyperhermitian with respect to $\left\{J_{\alpha}\right\}_{\alpha=1,2}$ and therefore there exists a positive differentiable function $\rho$ on $G$ such that $\tilde{\phi}^{*}\langle,\rangle^{x y}=\rho\langle$,$\rangle . Since \tilde{\phi}$ is an automorphism, the pull-back $\tilde{\phi}^{*}\langle,\rangle^{x y}$ is also left invariant; hence $\rho$ must be constant on $G$.

By assumption, $\langle,\rangle^{\prime}$ is hyperhermitian with respect to $\left\{J_{\alpha}^{0}\right\}_{\alpha=1,2}$; hence $J_{z}^{0}$ is $\langle,\rangle^{\prime}$-orthogonal for all $z \in S^{2}$. Therefore, $\langle,\rangle^{\prime}$ is hyperhermitian with respect to $\left\{J_{x}^{0}, J_{y}^{0}\right\}$ and, again, since $\langle,\rangle^{\prime}$ and $\langle,\rangle^{x y}$ are left invariant hyperhermitian metrics relative to the same structure, there is a positive real number $\beta$ such that $\langle,\rangle^{\prime}=$ $\beta\langle,\rangle^{x y}$. Now the proposition follows by taking $\lambda=\rho \beta$ and $\tilde{\phi}$.

The next step is to obtain the invariant hyperhermitian metrics. It turns out that there are five different metrics, up to homotheties, as the following theorem shows.

Theorem 4.2. If $\langle$,$\rangle is an invariant hyperhermitian metric on G$ then there exists a positive real number $\lambda$ such that $(G, \lambda\langle\rangle$,$) is isometric to one of the following$ riemannian spaces:

(i) $\mathbb{R}^{4}$ with the euclidean metric;

(ii) the riemannian product $\mathbb{R} \times S^{3}$ with the canonical metrics;

(iii) the riemannian product $\mathbb{R} \times \mathbb{R} H^{3}$, where $\mathbb{R} H^{3}$ has the symmetric metric of negative sectional curvature;

(iv) $\mathbb{R} H^{4}$ with the symmetric metric;

(v) $\mathbb{R}^{4}$ with a homogeneous non-symmetric metric of negative sectional curvature.

Moreover, 〈, > determines $G$ up to isomorphism.

Proof. If $\mathfrak{g}$ denotes the Lie algebra of $G$, by the results of Section 3 we have the following possibilities for $\mathfrak{g}$ : (i) $\mathfrak{g}$ is abelian, (ii) $\mathfrak{g} \cong \mathbb{R} \oplus \mathfrak{s o}(3)$, (iii) $\mathfrak{g} \cong \mathfrak{a f f}(\mathbb{C})$, (iv) $\mathfrak{g}$ is the solvable Lie algebra corresponding to $\mathbb{R} H^{4}$ or (v) $\mathfrak{g}$ is the solvable Lie algebra corresponding to $\mathbb{C} H^{2}$.

If (i) holds then $G$ is isomorphic to $\left(\mathbb{R}^{4},+\right)$. By Proposition 4.1 , we may assume that $\left\{J_{\alpha}\right\}_{\alpha=1,2}$ is given by

$$
\begin{array}{lll}
J_{1} Z=X, & J_{1} Y=W, & J_{1}^{2}=-I, \\
J_{2} Z=Y, & J_{2} W=X, & J_{2}^{2}=-I,
\end{array}
$$

in a basis $\{X, Y, Z, W\}$ of $\mathfrak{g}=T_{0} \mathbb{R}^{4}$, and that $\langle$,$\rangle is obtained by left translating$ the inner product such that this basis is orthonormal. Thus $\langle$,$\rangle is the canonical$ metric on $\mathbb{R}^{4}$.

If (ii) holds then $G$ is isomorphic to $\mathbb{R} \times S^{3}$. Again, by Proposition 4.1, we may assume that $\left\{J_{\alpha}\right\}_{\alpha=1,2}$ is as follows:

$$
\begin{array}{lll}
J_{1} Z=X, & J_{1} Y=W, & J_{1}^{2}=-I, \\
J_{2} Z=Y, & J_{2} W=X, & J_{2}^{2}=-I,
\end{array}
$$


where $\{Z, X, Y, W\}$ is as in Theorem 3.1, and that $\langle$,$\rangle is obtained by left translating$ the inner product such that this basis is orthonormal. The Lie subgroups of $G$ corresponding to $\mathbb{R} Z$ and $\operatorname{span}\{X, Y, W\}$ are $\mathbb{R}$ and $S^{3}$, respectively. From [2] (Lemma 4.1) it follows that $(G,\langle\rangle$,$) is isometric to the riemannian product \mathbb{R} \times S^{3}$ with the metric induced by $\langle$,$\rangle on each factor. Note that \langle$,$\rangle induces on S^{3}$ the canonical metric with constant sectional curvature 1.

If (iii) holds then $\mathfrak{g}$ admits a basis $\{X, Y, Z, W\}$ as in Theorem 3.3. By Proposition 4.1 we may assume that $\left\{J_{\alpha}\right\}_{\alpha=1,2}$ is defined by

$$
\begin{array}{lll}
J_{1} X=-W, & J_{1} Y=Z, & J_{1}^{2}=-I, \\
J_{2} X=Y, & J_{2} Z=-W, & J_{2}^{2}=-I,
\end{array}
$$

and that $\langle$,$\rangle is obtained by left translating the inner product with respect to which$ the above basis is orthonormal. Let us denote by $T$ and $K$ the Lie subgroups of $G$ with Lie algebras $\operatorname{span}\{X, Y, Z\}$ and $\mathbb{R} W$, respectively. Note that $a d_{W \mid \operatorname{span}\{X, Y, Z\}}$ is skew-symmetric, and, again, by [2] (Lemma 4.1), we obtain that $(G,\langle$,$\rangle ) is$ isometric to the riemannian product $T \times K$ with the metric induced by $\langle$,$\rangle on$ each factor. Both, $T$ and $K$, must be simply connected; therefore $T$ is the simply connected solvable Lie group corresponding to $\mathbb{R} H^{3}$ and $K=\mathbb{R}$. Note that $\langle$, induces on $T$ the left invariant metric that makes $T$ isometric to $\mathbb{R} H^{3}$ (cf. [3]).

If (iv) holds then $\mathfrak{g}$ admits a basis $\{A, X, Y, Z\}$ as in part (a) of Theorem 3.4. By Proposition 4.1 we may assume that $\left\{J_{\alpha}\right\}_{\alpha=1,2}$ is given by

$$
\begin{array}{lll}
J_{1} A=X, & J_{1} Y=Z, & J_{1}^{2}=-I, \\
J_{2} A=Y, & J_{2} Z=X, & J_{2}^{2}=-I,
\end{array}
$$

and that $\langle$,$\rangle is the left invariant metric induced by the inner product whith respect$ to which the above basis is orthonormal. By results of $[3](G,\langle\rangle$,$) is isometric to$ the symmetric space $\mathbb{R} H^{4}$.

Finally, if (v) holds then $\mathfrak{g}$ admits a basis $\{A, X, Z, Y\}$ as in part (b) of Theorem 3.4. By Proposition 4.1 we may assume that $\left\{J_{\alpha}\right\}_{\alpha=1,2}$ is as follows:

$$
\begin{array}{lll}
J_{1} A=X, & J_{1} Y=Z, & J_{1}^{2}=-I, \\
J_{2} A=\frac{\sqrt{2}}{2} Z, & J_{2} X=\frac{\sqrt{2}}{2} Y, & J_{2}^{2}=-I,
\end{array}
$$

and that $\langle$,$\rangle is the left invariant metric induced by the inner product with or-$ thonormal basis $\left\{A, X, \frac{\sqrt{2}}{2} Z, \frac{\sqrt{2}}{2} Y\right\}$. $G$ is isomorphic to the solvable Lie group corresponding to the the complex hyperbolic space $\mathbb{C} H^{2}$; thus the underlying manifold structure on $G$ is the euclidean structure on $\mathbb{R}^{4}$. The metric $\langle$,$\rangle is not symmet-$ ric ([3], Proposition 3), and one obtains by direct calculation that it has negative sectional curvature.

The last assertion in the theorem follows from the fact that invariant hyperhermitian metrics on non-isomorphic Lie groups give rise to non-homothetic riemannian spaces.

Remark 4.1. The fact that a left invariant hyper-Kähler metric is flat together with Theorem 4.2 implies that, except for the euclidean metric, the invariant hyperhermitian metrics in dimension 4 are not hyper-Kählerian.

The solvable Lie algebra $\mathfrak{s}$ corresponding to the complex hyperbolic space $\mathbb{C} H^{2}$ (see Theorem 3.4 (b)) has a canonical complex structure $J$ given by $J A=X, J Z=$ 
$Y$ and $J^{2}=-I$. It is well known that the symmetric metric on $\mathbb{C} H^{2}$ is Kählerian with respect to $J$. It is easy to verify that this metric is hermitian, non-Kählerian with respect to the complex structure $J_{1}$ considered in (v) of Theorem 4.2 (equations (4.1)). Moreover, it is not hard to prove that $J_{1}$ is not of Kähler type; that is, there is no left invariant metric which is Kählerian with respect to $J_{1}$. In particular, $J_{1}$ is not equivalent to $J$ by an automorphism of $\mathfrak{s}$. Identifying $\mathbb{C} H^{2}$ with the open unit ball $D_{2}(\mathbb{C})=\left\{\left(z_{1}, z_{2}\right) \in \mathbb{C}^{2}: z_{1} \bar{z}_{1}+z_{2} \bar{z}_{2}<1\right\}$ (see [6]), the symmetric metric on $\mathbb{C} H^{2}$ corresponds to the Bergman metric on $D_{2}(\mathbb{C})$ and the complex structure $J$ corresponds to the canonical complex structure (inherited from $\mathbb{C}^{2}$ ) on $D_{2}(\mathbb{C})$. The complex structure $J_{1}$ can be transferred to $D_{2}(\mathbb{C})$ via the above identifications and therefore the Bergman metric is hermitian, non-Kählerian with respect to $J_{1}$. It follows from [5] that $D_{2}(\mathbb{C})$ with the Bergman metric is the only irreducible homogeneous bounded domain in $\mathbb{C}^{2}$ up to holomorphic isometries. This fact and the above observations imply:

Corollary 4.3. Every irreducible homogeneous bounded domain $D$ in $\mathbb{C}^{2}$ admits a complex structure $J_{1}$ (invariant by a solvable group of isometries) which is not equivalent (by a holomorphic isometry) to the canonical complex structure on D and such that the Bergman metric is hermitian, non-Kählerian with respect to $J_{1}$.

Using the geometric results obtained in this section we can conclude that the simply connected hypercomplex 4-manifolds arising from non-isomorphic Lie groups are not equivalent even by a hypercomplex diffeomorphism.

All the 4-dimensional simply connected Lie groups that admit invariant hypercomplex structures, other than $\mathbb{R} \times S^{3}$, are diffeomorphic to $\mathbb{R}^{4}$. This says that $\mathbb{R} \times S^{3}$ with its (unique) invariant hcs is not equivalent, by a hypercomplex diffeomorphism, to any of the remaining Lie groups with invariant $h c s$.

$\mathbb{R}^{4}$ with its (unique) invariant hcs is not equivalent to the simply connected solvable Lie group $S$ corresponding to the complex hyperbolic space $\mathbb{C} H^{2}$, with any of its invariant hcs. If they were equivalent, then the hypercomplex diffeomorphism would give a conformal equivalence between the hyperhermitian metrics, which by Theorem 4.2 are homothetic to (i) and (v), respectively. Therefore (v) would be conformally flat, a contradiction. In fact, an easy computation shows that the Weyl tensor of (v) does not vanish. The same argument shows that $S$ is not equivalent, as a hypercomplex manifold, to $\mathbb{R} \times \mathbb{R} H^{3}$ or $\mathbb{R} H^{4}$.

Finally, from a result of [7], one gets that $\mathbb{R}^{4}, \mathbb{R} H^{4}$ and $\mathbb{R} \times \mathbb{R} H^{3}$ belong to different conformal equivalence classes and therefore the corresponding groups with invariant $h c s$ are not equivalent by a hypercomplex diffeomorphism.

We can summarize the above observations as follows:

Corollary 4.4. Let $G$ and $G^{\prime}$ be simply connected 4-dimensional Lie groups with invariant hypercomplex structures $\left\{J_{\alpha}\right\}_{\alpha=1,2}$ and $\left\{J_{\alpha}^{\prime}\right\}_{\alpha=1,2}$, respectively. If there exists a hypercomplex diffeomorphism $f:\left(G,\left\{J_{\alpha}\right\}_{\alpha=1,2}\right) \rightarrow\left(G^{\prime},\left\{J_{\alpha}^{\prime}\right\}_{\alpha=1,2}\right)$ then $G$ and $G^{\prime}$ are isomorphic as Lie groups.

\section{REFERENCES}

1. C. P. Boyer, A note on hyperhermitian four-manifolds, Proc. Amer. Math. Soc. 102(1) (1988), 157-164. MR 89c:53049

2. M. J. Druetta, Visibility and rank one in homogeneous spaces of $K \leq 0$, Transact. Amer. Math. Soc. 304(1) (1987), 307-321. MR 88i:53084 
3. E. Heintze, On homogeneous manifolds of negative curvature, Math. Ann. 211 (1974), 23-34. MR 50:5695

4. D. Joyce, Compact hypercomplex and quaternionic manifolds, J. Differential Geom. 35 (1992), 743-761. MR 93g:53064

5. S. Kaneyuki \& T. Tsuji, Classification of homogeneous bounded domains of lower dimension, Nagoya Math. J. 53 (1974), 1-46. MR 50:5039

6. S. Kobayashi \& K. Nomizu, Foundations of differential geometry, vol II, Interscience, New York, 1963. MR 27:2945

7. T. Nagano, The conformal transformation on a space with parallel Ricci tensor, J. Math. Soc. Japan 11 (1) (1959), 10-14. MR 23:A1330

8. D. Snow, Invariant complex structures on reductive Lie groups, J. Reine Angew. Math. 371 (1986), 191-215. MR 87k:32058

9. J. E. Snow, Invariant complex structures on four-dimensional solvable real Lie groups, Manuscripta Math. 66 (1990), 397-412. MR 91c:32026

10. Ph. Spindel, A. Sevrin, W. Troost, \& A. Van Proeyen, Extended super-symmetric $\sigma$-models on group manifolds, Nuclear Phys. B 308 (1988), 662-698. MR 89m:53138

Famaf, Universidad Nacional de Córdoba, Ciudad Universitaria, 5000 - Córdoba, Argentina

E-mail address: barberis@mate.uncor.edu

Current address: Department of Mathematics, University of California at San Diego, La Jolla, California 92093

E-mail address: mbarberis@ucsd.edu 